\title{
Effect of the Vortex Formed by the Electrohydrodynamic Flow on the Motion of Particles in a Needle-plate Electrostatic Precipitator
}

\author{
Wenchao Gao ${ }^{1}$, Yifan Wang ${ }^{2}$, Hao Zhang ${ }^{2}$, Baoyu Guo ${ }^{1}$, Chenghang Zheng ${ }^{2}$, Jun Guo ${ }^{3}$, \\ Xiang $\mathbf{G a o}^{2^{*}}$, Aibing $\mathbf{Y u}^{1,4}$ \\ ${ }^{1}$ ARC Research Hub for Computational Particle Technology, Department of Chemical Engineering, Monash University, \\ Victoria 3800, Australia \\ ${ }^{2}$ State Key Lab of Clean Energy Utilization, State Environmental Protection Engineering Center for Coal-Fired Air \\ Pollution Control, Zhejiang University, Hangzhou 310027, China \\ ${ }^{3}$ Fujian Longking Co., Ltd., Longyan 364000, China \\ ${ }^{4}$ Southeast University-Monash University Joint Research Institute, Suzhou Industrial Park, Jiangsu 215100, China
}

\begin{abstract}
This work numerically simulates the effect of the electrodynamic (EHD) flow on particle motion in a single-needle-plate electrode configuration. The interaction between the primary-secondary flow, and the trajectory of particles in a 3D environment is analyzed. In addition, the effects of the needle-shaped discharge electrode structure on the electric field and the flow field distribution are explored. The results show that the sharp tip of the needle emits a high-intensity discharge that generates a nearby high-speed ionic wind, which can reach a velocity of $9.028 \mathrm{~m} \mathrm{~s}^{-1}$ at an applied voltage and an inlet velocity of $-60 \mathrm{kV}$ and $1 \mathrm{~m} \mathrm{~s}^{-1}$, respectively. This ionic wind near the needle tip potentially increases the migration speed of particles. Moreover, $90 \%$ of the $1 \mu \mathrm{m}$ particles penetrate the surface of the outlet, indicating that the EHD flow negatively affects the capture of fine particles. The relationships between the injection position, the residence time, and the escape velocity of the particles further confirm that the secondary flow significantly inhibits fine-particle capture. These findings can be applied to optimize an electrode design that efficiently uses high-speed ionic wind to capture particles, including the fine fraction.
\end{abstract}

Keywords: Electrostatic precipitator; Electrohydrodynamic flow; Needle discharge electrode; Vortex; Particle motion.

\section{NOMENCLATURE}

$\varphi \quad$ Electric potential

$\varepsilon_{0} \quad$ Permittivity of free space

$k_{\text {ion }}$ Mobility of ion

$D_{e} \quad$ Diffusion coefficient

$\vec{J} \quad$ Current density

$\rho_{\text {ion }}$ Ion charge density

$\vec{E} \quad$ Electric field strength

$\overrightarrow{u_{g}}$ Gas velocity

$E_{s} \quad$ Corona onset electric strength

$E_{0} \quad$ Breakdown electric field strength

$m$ Dimensionless surface parameter

$\delta \quad$ Relative density

$r \quad$ Radius of discharge electrode

\footnotetext{
* Corresponding author.

Tel.: +86-571-87951335; Fax: +86-571-87951616

E-mail address: xgao1@zju.edu.cn
}

$\begin{array}{ll}\rho & \text { Mass density of the gas } \\ P & \text { Absolute pressure } \\ \mu & \text { Dynamic viscosity of the gas } \\ m_{p} & \text { Particle mass } \\ \overrightarrow{u_{p}} & \text { Particle velociuty } \\ C_{d} & \text { Drag coefficient } \\ \operatorname{Re}_{p} & \text { Particle Reynolds number } \\ C_{c} & \text { Cunningham correction factor } \\ \lambda & \text { Mean free path of the gas } \\ q_{s} & \text { Saturation charge } \\ \varepsilon_{r} & \text { Relative permittivity of the particles } \\ \tau & \text { Time constant of particle charging } \\ k_{B} & \text { Boltzmann constant } \\ E & \text { Unit electronic charge }\end{array}$

\section{INTRODUCTION}

Electrostatic precipitators are used in various industries to remove fly ash from emissions (Jaworek et al., 2018; Zheng et al., 2019). Countries have raised the emission standards of particulate matter, such as strict $\mathrm{PM}_{2.5}$ ultralow emission standard, due to the deterioration of air pollution (Tang et 
al., 2019). The mass collection efficiency of many types of electrostatic precipitators (ESPs) has exceeded 99\% (Dong et al., 2018). As such, the capture efficiency of submicron particles may be further improved (Guo et al., 2014a; Guo et al., 2015; Jaworek et al., 2018; Zhu et al., 2019; Yang et al., 2020; Zheng et al., 2020a, b). The basic principle of the ESP operation is that particles are charged, and the particleladen gas flows through the region where an electric field is applied to make the particles migrate to the collection plate (Czech et al., 2012; Adamiak, 2013; Guo et al., 2013; Yang et al., 2019). In this process, the ionization of air molecules around the thinnest electrode can cause a corona discharge. These ions are subject to the Coulomb force due to the action of the electric field, causing them to move from the active electrode to the grounded collection plate. Therefore, many collisions in motion are present between ions and neutral air molecules, thereby leading to momentum transfer and airflow (also called electrohydrodynamic [EHD] flow) (Soldati, 2000; Wang et al., 2019). A phenomenon called ionic wind is found in some localized areas when a high-intensity voltage is applied to a sharp electrode surface (Park et al., 2018). Thus, observing how the flow field affects the movement of micron-sized particles in ESP is necessary.

Many studies on EHD flow have been published. Niewulis et al. (2009), Podlinski et al. (2009) and Niewulis et al. (2013) have established a particle image velocimetry (PIV) experiment to observe the flow velocity fields in narrow electrostatic precipitators, but this experiment shows the distribution of field flow and streamlines at different voltages and does not explain how EHD flow affects particle motion. Another EHD experimental study has been conducted to collect low-resistive diesel particulates in different orientations of discharge electrodes (Kawakami et al., 2013). The dust collection efficiency under different particles is measured experimentally, and the influence of the special electrode structure on the flow field is not described in detail. A previous report has indicated that the geometry of the discharge electrode is one of the important factors of ionic wind distribution, but the analysis of how the electrode structure affects the efficiency is lacking (Wang et al., 2017). Recently, Moreau et al. (2018) have built a PIV experiment with fast-gateable intensifiedCCD (ICCD) camera and proposed that the ionic wind produced by negative and positive corona discharges is different in air without added particles. Nevertheless, showing the particle motion behavior after being affected by EHD flow in these experiments remains difficult. In addition, experimental studies require a huge cost and a long time especially when a large-scale platform needs to be built.

Some numerical simulation studies have investigated the effect of EHD flow on ESP performance. In the early reports, a 2D wire-plate-type electrostatic precipitator is modeled numerically by Chun et al. $(2006,2007)$ to simulate the flow velocity fields close to the discharge electrode. In this work, the computational fluid dynamics method with the $k-\varepsilon$ turbulent model is involved. Some simulation results show that the influence of the geometry of discharge electrode and collection plate on the distribution of EHD flow field is different (Feng et al., 2018; Shen et al., 2018). However, these studies have only investigated the flow field with the sample structure of electrodes (such as wire electrode) in a 2D environment. The analysis of particle motion behavior has not been proposed in a spatial state. The 3D numerical analysis of EHD flow in a spiked electrode-plate ESP has been conducted by some research groups (Fujishima et al., 2004, 2006; Farnoosh et al., 2010, 2011; Dong et al., 2019). Fujishima et al. (2006) have described flow interaction by using the dimensionless number $N_{E H D}$ and the relative distance between the spike point spacing and the wire-to-plate spacing $\left(S_{z} / d\right)$. The EHD secondary flow pattern and its interaction with the inlet flow in different planes with various applied voltages have been observed by Farnoosh et al. (2011). Although the above studies have improved the understanding of the flow field in the ESP, most of them are limited to the single geometry of the spike-plate-type discharge electrode. In addition, the influence of ionic wind on the process of particle capture (e.g., particle trajectory and particle size) has not been considered in detail.

The needle discharge electrode presented in our previous work can contribute to the complexity of the corona electrostatic and flow fields (Guo et al., 2013, 2014b; Wang et al., 2019). In the present study, a single-needle corona wire with flat collection plate is established to determine the effect of EHD flow on the particle motion behavior with different particle sizes. For the first time, the results of numerical simulation show the multiple relationships among the particle injection position, escape velocity, and residence time. The findings can provide the basis for the optimal design of ESP and the reasonable utilization of vortex generated by EHD to improve the efficiency of particle collection.

\section{NUMERICAL METHOD}

\section{Numerical Algorithm}

In this study, the computational model of the needle electrode-plate ESP involves corona discharge, gas flow, particle charge, and particle motion, which are all calculated using computational fluid dynamics. The major governing equations for simulated electric field, gas flow, particle trajectory, and particle charging process are also provided (Guo et al., 2014b; Gao et al., 2020a, b).

The Poisson equation for electrical potential $(\varphi)$ and the current continuity equation are as follows:

$$
\begin{aligned}
& \nabla^{2} \varphi=-\frac{\rho_{\text {ion }}}{\varepsilon_{0}} \\
& \nabla\left(\rho_{\text {ion }}\left(k_{\text {ion }} \vec{E}+\overrightarrow{u_{g}}\right)-D_{e} \nabla \rho_{\text {ion }}\right)=0
\end{aligned}
$$

and

$\vec{E}=-\nabla \varphi$

The corona current can be calculated as follows:

$\vec{J}=\rho_{\text {ion }}\left(k_{\text {ion }} \vec{E}+\overrightarrow{u_{g}}\right)-D_{e} \nabla \rho_{\text {ion }}$ 
The corona onset electric strength $\left(E_{s}\right)$ is calculated in accordance with Peek's law on the surface of discharge electrode:

$$
E_{s}=E_{0} m\left(\delta+0.0308 \sqrt{\frac{\delta}{r}}\right)
$$

The particle flow in the ESP can be considered as state turbulence, and the RNG $k-\varepsilon$ model can be used to describe the steady-state turbulent flow. The conservation of mass (Eq. (6)) and momentum (Eq. (7)) for the gas flow are as follows:

$$
\nabla \cdot\left(\rho \overrightarrow{u_{g}}\right)=0
$$

and

$$
\rho\left[\frac{\partial \overrightarrow{u_{g}}}{\partial t}+\overrightarrow{u_{g}} \cdot \nabla \overrightarrow{u_{g}}\right]=-\nabla \vec{P}+\mu \nabla^{2} \overrightarrow{u_{g}}+\rho_{i o n} \vec{E}
$$

In the Lagrange framework, the discrete element method is used to track the suspended particles entrained in the gas phase. The equation of particle motion can be described as:

$$
m_{p} \frac{d \overrightarrow{u_{p}}}{d t}=\frac{1}{8} \pi C_{d} \rho d_{p}^{2}\left|\vec{u}-\overrightarrow{u_{p}}\right|\left(\vec{u}-\overrightarrow{u_{p}}\right)+q_{p} \vec{E}
$$

where

$$
C_{d}=\max \left[\frac{24}{C_{c} \operatorname{Re} e_{p}}\left(1+0.15 R e_{p}^{0.687}\right), 0.44\right]
$$

and

$$
C_{c}=1+\frac{2 \lambda}{d_{p}}\left(1.257+0.4 e^{-1.1 d_{p} / 2 \lambda}\right)
$$

Particle charging is an important process that determines the particle behavior. The dimensionless and lawless charging equations are used as follows:

$$
\frac{d q_{p}}{d t}= \begin{cases}\frac{q_{s}}{\tau}\left(1-\frac{q}{q_{s}}\right)^{2}+\frac{2 \pi \alpha \rho k_{i o n} k_{B} d_{p}}{e} & q<q_{s} \\ \frac{\alpha}{4 \tau}\left(q-q_{s}\right) \exp \left(\frac{e+\left(q_{s}-q\right)}{2 \pi \varepsilon_{0} k_{i o n} k_{B} T d_{p}}\right) & q>q_{s}\end{cases}
$$

and

$$
\alpha= \begin{cases}1 & e_{\text {norm }}<0.525 \\ \frac{1}{\left(e_{\text {norm }}+0.457\right)^{0.575}} & e_{\text {norm }}>0.525\end{cases}
$$

The number of dimensionless charging in the above equations are defined as:

$$
\begin{aligned}
& e_{\text {norm }}=\frac{e d_{p}}{2 k_{B} T} E \\
& \tau=\frac{4 \varepsilon_{0}}{\rho_{\text {ion }} k_{\text {ion }}}
\end{aligned}
$$

and

$$
q_{s}=3 \frac{\varepsilon_{r}}{\varepsilon_{r}+2} \pi \varepsilon_{0} d_{p}^{2} E
$$

\section{MODEL DESCRIPTION}

The investigated ESP model consists of two flat and parallel collecting plates, which are electrically conducting and grounded, and a single-needle-type corona wire installed in the front of the channel (Fig. 1). The ESP channel is 960 $\mathrm{mm}$ long, $400 \mathrm{~mm}$ wide, and $200 \mathrm{~mm}$ high (same as the discharge electrode). The appearance of the corona wire is shown in Fig. 1(b), and the detailed dimensions of the needle body and needle tip are shown in Figs. 1(c) and 1(d). The whole geometry is discretized using a structured mesh, and refined grids are applied around the needles to improve the computational accuracy during the discharge process. The other conditions are as follows:

- Gas: inlet velocity, $1 \mathrm{~m} \mathrm{~s}^{-1}$; gas density, $1.093 \mathrm{~kg} \mathrm{~m}^{-3}$; and temperature, $323.15 \mathrm{~K}$.

- Molecules and gaseous ions: molecular mean free path, $6.8 \times 10^{-8} \mathrm{~m}$; electronic charge, $1.6 \times 10^{-19} \mathrm{C}$; and ion mobility, $2.1 \times 10^{-4} \mathrm{~m}^{2}(\mathrm{~V} \cdot \mathrm{s})^{-1}$.

- Particles: particle inlet velocity, $1 \mathrm{~m} \mathrm{~s}^{-1}$; mass density, $2100 \mathrm{~kg} \mathrm{~m}^{-3}$; diameter, $1-30 \mu \mathrm{m}$; and relative permittivity, 10.

- The applied voltage on the needle electrode is $-60 \mathrm{kV}$, and the plate side is electrically grounded with zero voltage.

The needle structure electrode is widely used in ESP because of its high-intensity partial discharge characteristics and high flow velocity. Some studies prove these phenomena, and their simulation results agree well with our experimental data (Guo et al., 2014b; Wang et al., 2019). For example, the important quantitative analysis is conducted using numerical experiments in terms of some significant electric characteristics on the plate as the applied voltage changes. Also, the numerical data are compared with experimental data from our industrial-scale ESP to prove the accuracy. In addition, the investigated model has been validated in previous studies, and the discharge performance of the needle electrode is shown in detail, where the current density generated around the blunt electrode is much lower than that of the sharp electrode (Guo et al., 2013, 2014a, b, 2015, 2017; Yang et al., 2019). The present study aims to observe the effect of secondary flow on particle motion in electrostatic field. However, the simulation process only involves a single corona wire because the focus is the trajectory of particles 

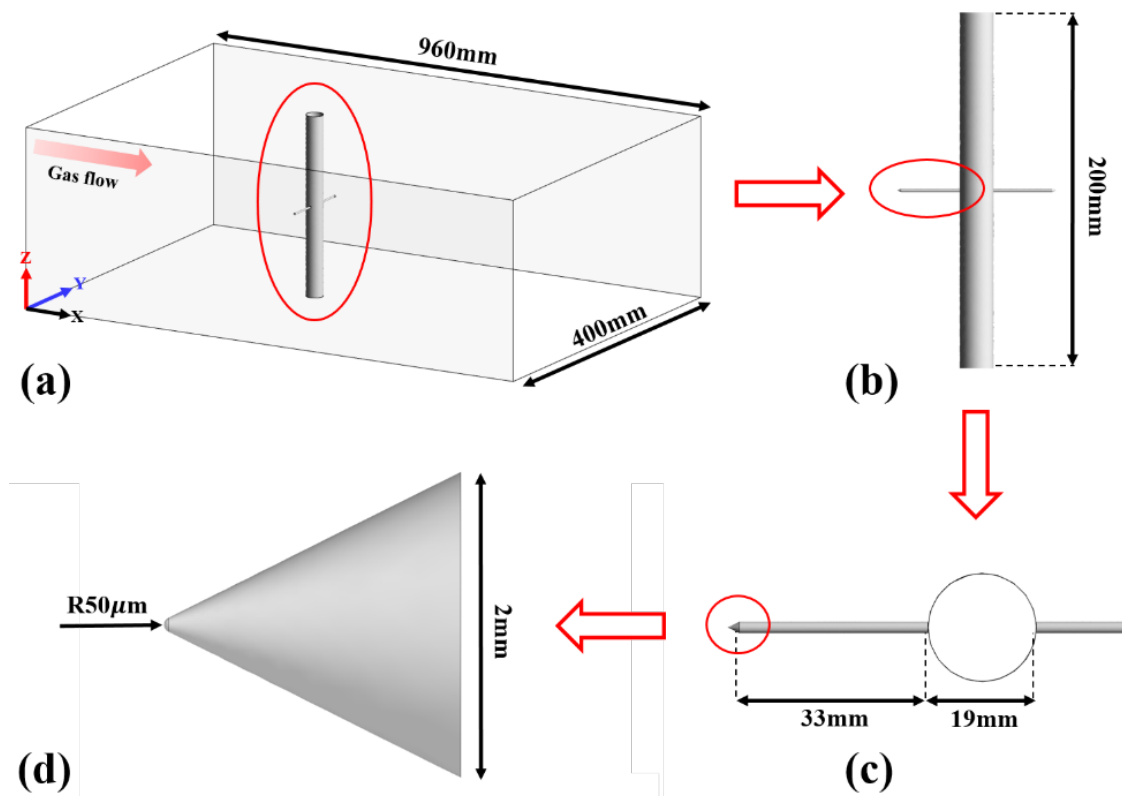

(b)

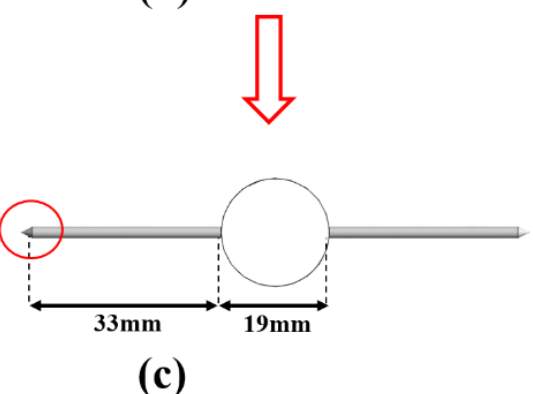

Fig. 1. Schematic of simulation domain and dimensions.

passing through the discharge electrode. Thus, the simulated collection efficiency of particles is not high compared with that of multiple-discharge electrode configurations.

\section{NUMERICAL RESULTS}

\section{Electrical Characteristics}

The distribution of electric field and potential on different direction plates is shown in Fig. 2. The applied voltage has remained constant at $-60 \mathrm{kV}$ in the whole simulation process. Different Peek's formulas are applied on four different discharge surfaces, namely, wire surface, needle body, tip body, and needle tip, because the discharge electrode is not a simple circular wire, and the curvature radius of the faces is not consistent. Results show that the highest field strength of $4.095 \times 10^{6} \mathrm{~V} \mathrm{~m}^{-1}$ is observed at the needle tip, which has a radius of only $50 \mu \mathrm{m}$. In these three planes, the electric field strength and $\varphi$ along the direction of discharge electrode decrease sharply. The results of the electric field distribution in the $Y Z$ plane demonstrate that the electric field strength decreases in the special region near where the angle is formed by the main part of the electrode wire and needle body due to corona suppression. The electric potential near the collecting plate is lower than that in other regions. Thus, the distribution of electrical characteristics demonstrates that the particles being injected far away from the discharge electrode or entering the corona suppression area may be unfavorable for collection.

\section{Flow Velocity Distribution of Needle-plate ESP in 3D Space}

Gas flow direction and velocity play crucial roles in particle trapping. On the one hand, a suitable inlet velocity needs to be set for injecting dust flow. If the inlet velocity is too fast, the particles become insufficiently charged, and the slow velocity causes dust deposition in the ESP channel. In this study, the inlet velocity is set to three different flow rates $\left(0.5,0.75\right.$, and $\left.1 \mathrm{~m} \mathrm{~s}^{-1}\right)$. On the other hand, the effect of EHD flow generated during the discharge process on particle movement cannot be avoided. Therefore, the analysis should be performed in $3 \mathrm{D}$ when studying the internal flow field of ESP.

The flow velocity distributions in $X Y, X Z$, and $Y Z$ direction planes under different inlet velocities are shown in Fig. 3. The results in Fig. 2 show that the electric field strength around the needle tip of the electrode is stronger than those in other areas. Moreover, the maximum velocity of ionic wind can reach $9.028 \mathrm{~m} \mathrm{~s}^{-1}$ under the applied voltage of $-60 \mathrm{kV}$ at $1 \mathrm{~m} \mathrm{~s}^{-1}$ inlet speed and rapidly decreases with increased distance away from the needle tip, as shown in the enlarged drawing. Two high-speed areas are created on both sides of the needle electrode due to the vertical relationship between the gas flow direction and the needle tip direction, as shown in the $X Y$ plane. As the inlet velocity increases from $0.5 \mathrm{~m} \mathrm{~s}^{-1}$ to $1 \mathrm{~m} \mathrm{~s}^{-1}$, the position of the two high-speed regions also shifts inward. Besides, the velocity distribution is likely to be uniform on the $Y Z$ plane with the increase in inlet velocity. Finally, a low-speed zone is found clearly at the rear end of the main body of the discharge electrode in the $X Y$ and the $X Z$ planes at a velocity close to $0 \mathrm{~m} \mathrm{~s}^{-1}$ because the movement of the airflow is blocked by the electrode body. The range of the low-speed area increases as the inlet flow rate decreases from $1 \mathrm{~m} \mathrm{~s}^{-1}$ to $0.5 \mathrm{~m} \mathrm{~s}^{-1}$. This result demonstrates that the distribution of flow field velocity is significantly affected by the high-speed ionic wind and that the direction of the EHD flow depends on the direction of the strongest discharge point. The high-speed ionic wind pushes the particles moving near the tip of the needle to the collecting plate, which is conducive to collecting dust. The particle movement may become unpredictable as the particles pass through the low-speed zone behind the discharge electrode. After the comparison of the $3 \mathrm{D}$ flow field distribution 
Electric field $\left(\mathrm{V} / \mathrm{m} \times \mathbf{1 0}^{6}\right)$

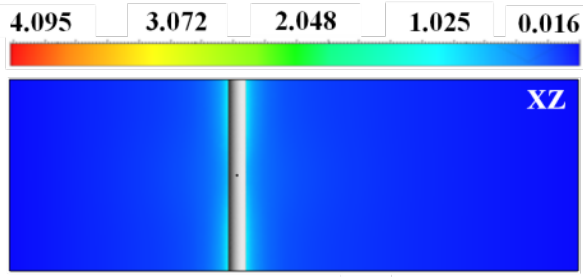

\section{Electric potential (-kV)}

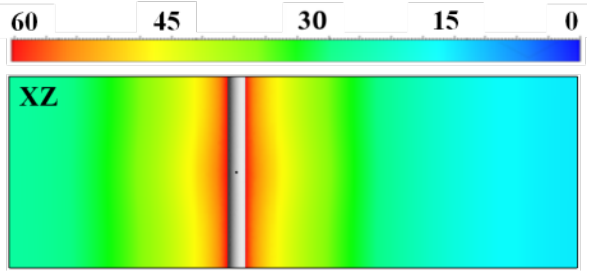
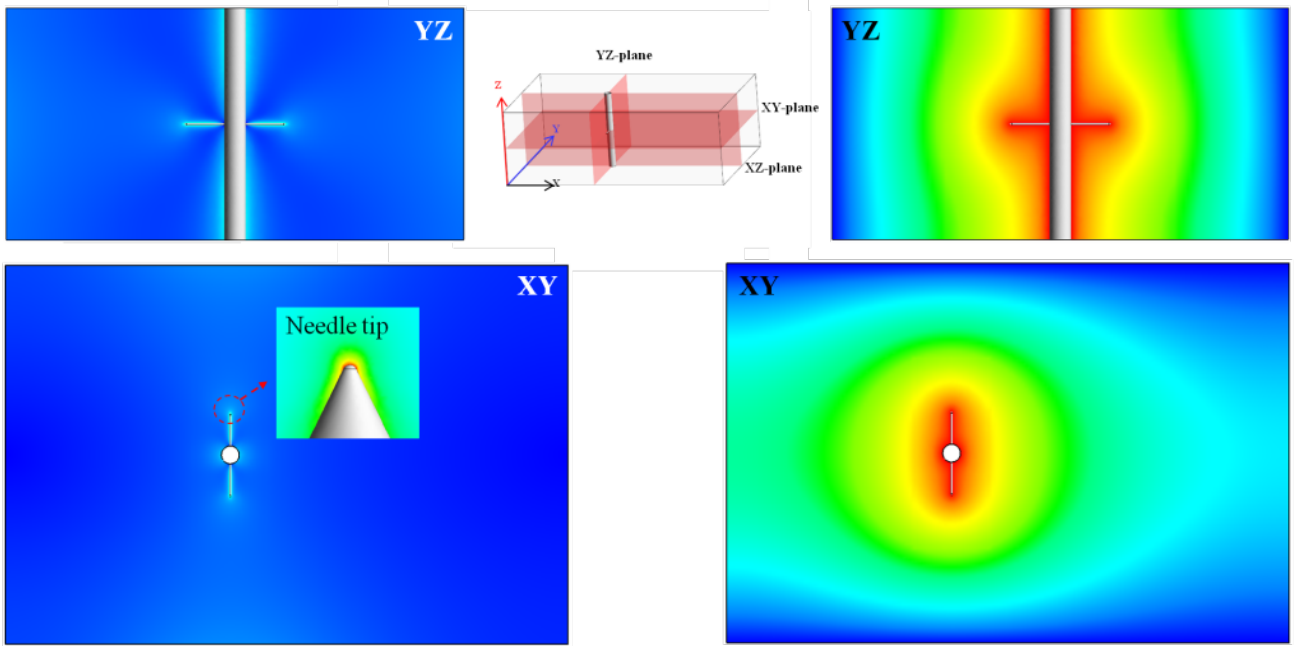

Fig. 2. Distribution of electric field and electric potential in three direction planes.
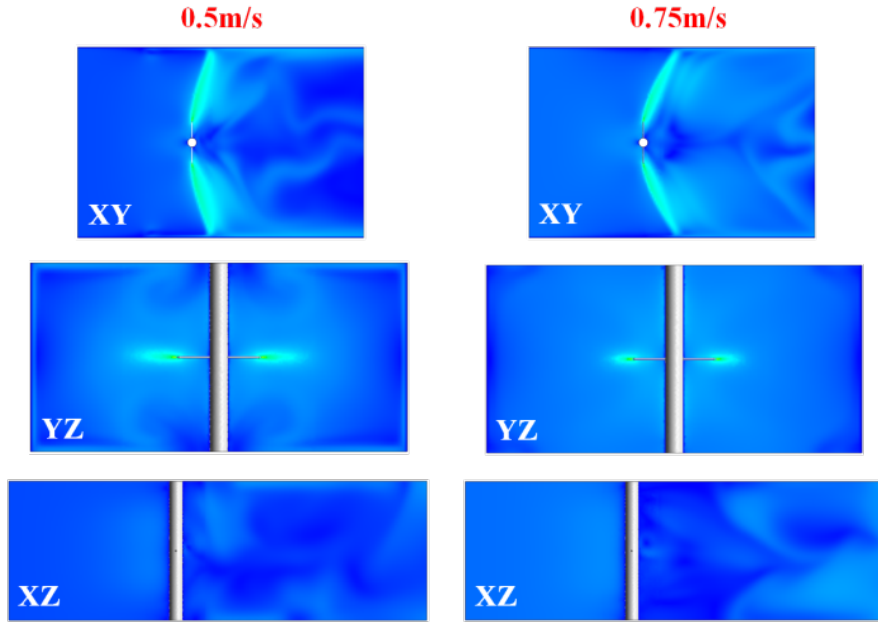

$0.75 \mathrm{~m} / \mathrm{s}$
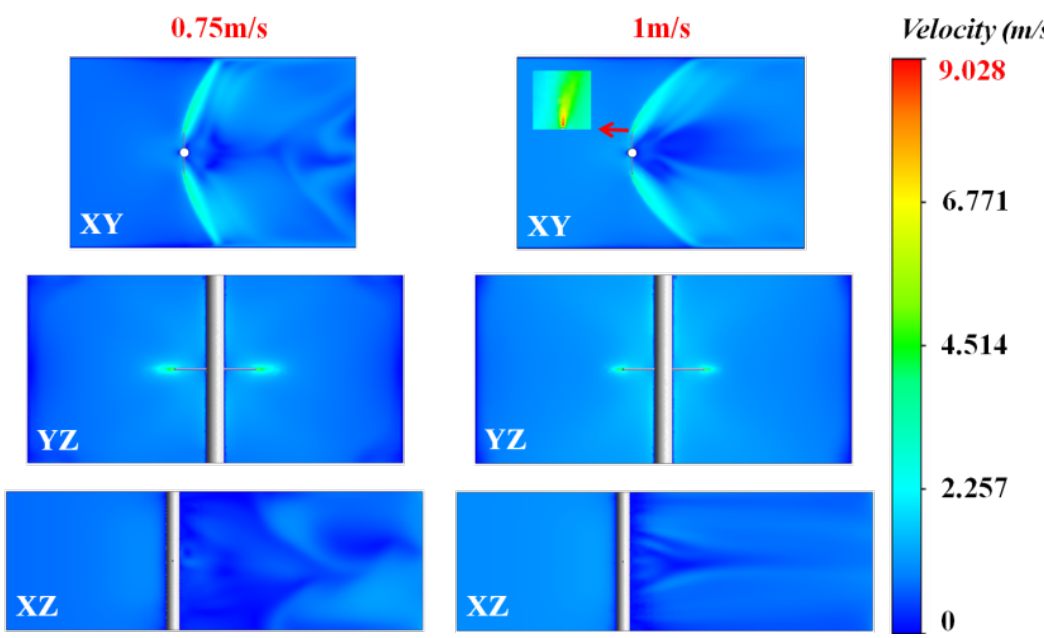

Fig. 3. Flow velocity distributions in $X Y, Y Z$, and $X Z$ direction planes under different inlet velocities.

under different inlet velocities, an inlet velocity of $1 \mathrm{~m} \mathrm{~s}^{-1}$ is used as the basic simulation condition because the position of the high-speed region at the needle tip may help the particles move easily toward the collection plate at an inlet flow velocity of $0.5 \mathrm{~m} \mathrm{~s}^{-1}$. However, the low-velocity zone formed by the low inlet velocity has large size and complex distribution, which are not conducive to particle capture especially when dealing with high-concentration flue gas.

Results show that the velocity distribution is perpendicular to the ESP channel in Planes A-I with an applied voltage of $-60 \mathrm{kV}$ and spaced uniformly from $0 \mathrm{~mm}$ in the middle of the needle discharge electrode $(x=0 \mathrm{~mm})$ to $36 \mathrm{~mm}$ at the end of the ESP channel $(x=+36 \mathrm{~mm})$, as shown in Fig. 4 .
The velocity at the rear end of the corona wire is lower than that in other regions, and the interference of the discharge electrode is significantly reduced as the plane moves from $B$ to I. Planes B-I evidently show that the distribution of the low-speed region depends on the structure of the discharge electrode. In Plane A, the flow velocity around the electrode is apparently higher than that in other areas far from the corona wire, especially at the two needle tips. This finding may be because the strength of ionic wind depends on ion charge density and electric field strength. Thus, the corona discharge around the needle tip is an important element for generating ionic wind with high velocity. After the gas flow passes through the needle electrode, the velocity distribution 

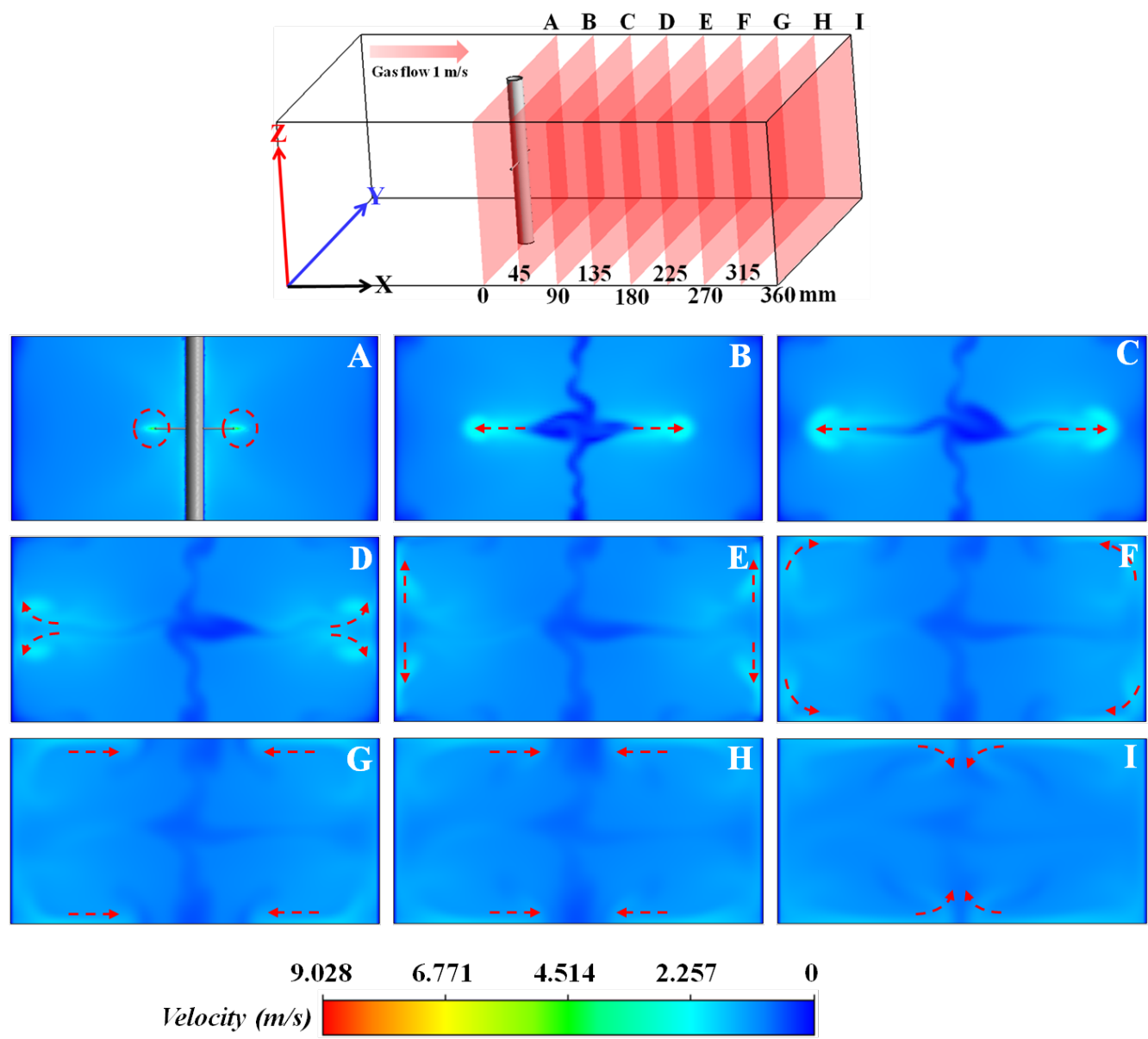

Fig. 4. Velocity distributions in Planes A-I with $-60 \mathrm{kV}$ applied voltage and $1 \mathrm{~m} \mathrm{~s}^{-1}$ inlet velocity.

in Plane B shows that the two spherical flows are emitted from the tip of the needle and that the evolution of EHD flow is similar to that of two mushroom clouds moving toward the collection plate, as shown in Plane C. The result from Planes D-I reveals that the high-speed ionic wind is divided into two small flows after hitting the collection surface and then induced back to the middle and back ends of the ESP channel. The red arrows in these figures indicate the direction and the trajectory of the high-speed ionic wind. This important result highlights the effect of primary inlet flow and needle electrode structure on the secondary EHD flow. This high-speed swirling motion of the secondary flow may adversely affect the collection efficiency due to the particles being entrapped to the EHD flow.

Fig. 5 illustrates the pronounced effect of the ionic wind on the velocity streamlines. The six planes are named $A-F$, where Planes A, B, and $\mathrm{C}$ are perpendicular to the ESP channel and spaced uniformly from $0 \mathrm{~mm}$ in the middle of the needle discharge electrode $(x=0 \mathrm{~mm})$ to $360 \mathrm{~mm}$ at the end of the ESP channel $(x=360 \mathrm{~mm})$. Two pairs of vortices located in the upper and the lower areas of the needle tip are generated in Plane A due to the strong interaction between the inlet flow and the secondary EHD flow. When the inlet flow passes through the needle electrode, most of the space that the main airflow passes through is blocked by these four vortices. The streamline clearly shows that the airflow produces high-speed ionic wind at the tip of the needle and leads back to the center of the channel by colliding with the collection surface. These four vortices do not debilitate as airflows pass through Planes B and C. A low-speed region exists at the back of the discharge electrode in Planes A-C. Thus, Planes D-F are horizontal to the flow direction and spaced uniformly from $0 \mathrm{~mm}$ in the middle of the corona wire $(y=0 \mathrm{~mm})$ to $40 \mathrm{~mm}$ at the left side of the wire to observe the airflow movement at the rear end of the discharge wire (Fig. 6). In Planes D and E, the number of vortices increases from one to two as the distance from the center of the electrode becomes shorter. In Plane F, the airflow behind the wire is very chaotic and has several vortices, but its size is far less than those of four large vortices presented in Planes A-C. This result demonstrates that vortex formation is closely related to the geometry of the discharge electrode and collection plate. In this case, the phenomenon of four symmetrical vortices formed by the needle structure electrode and the high-intensity electric strength may change the movement behavior of particles. A nonflat dust-collecting plate may help reduce vortex generation when high-speed ionic wind hits the dust-collecting pole. Aside from the four large-sized vortices, the influence of small-sized eddy currents existing behind the cylindrical shape of the corona wire and needle body on particle motion should be the focus when determining the effect of the particle trajectory. 

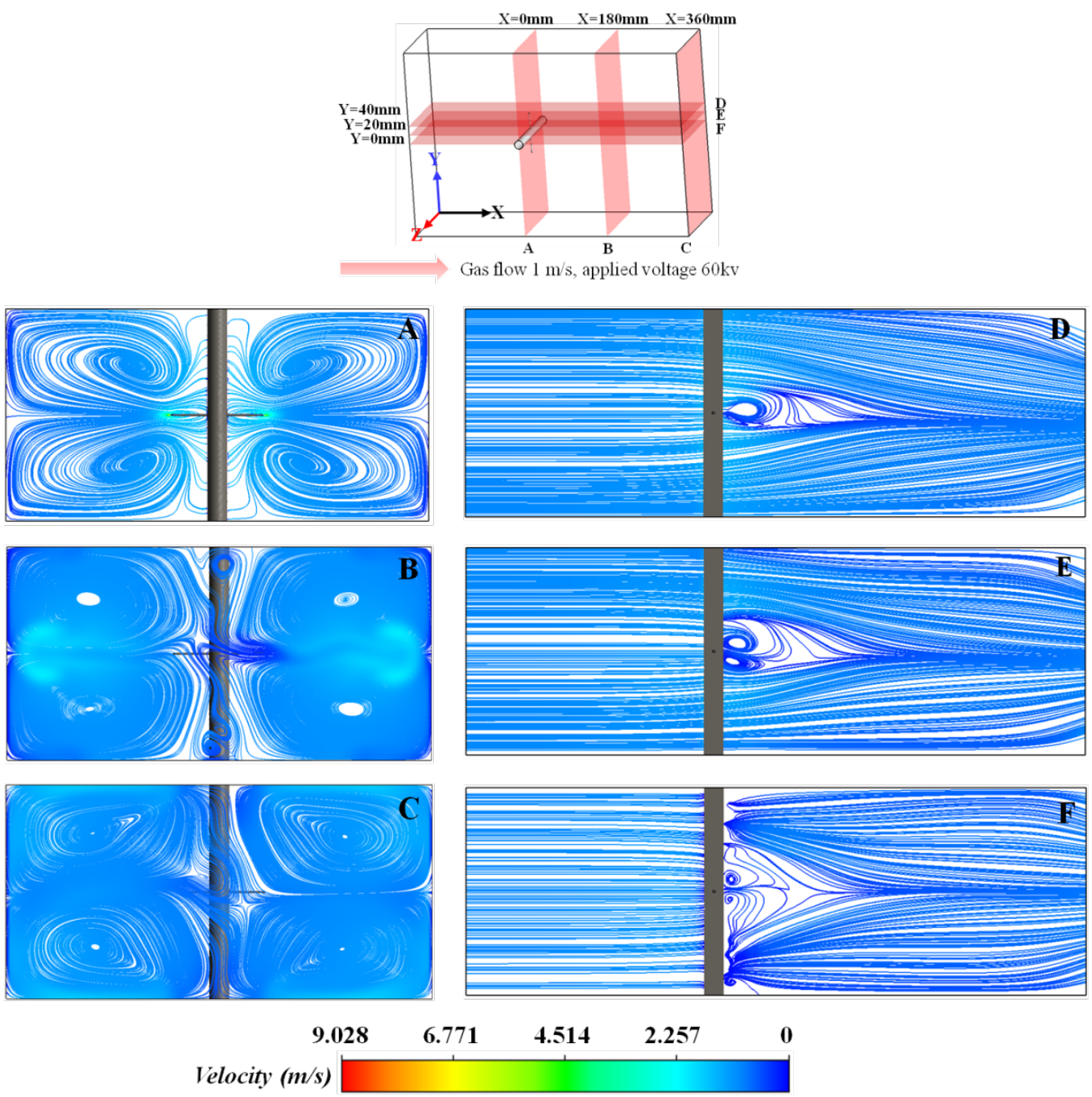

Fig. 5. Secondary EHD flow streamlines in Planes A-F at applied voltage of $-60 \mathrm{kV}$.
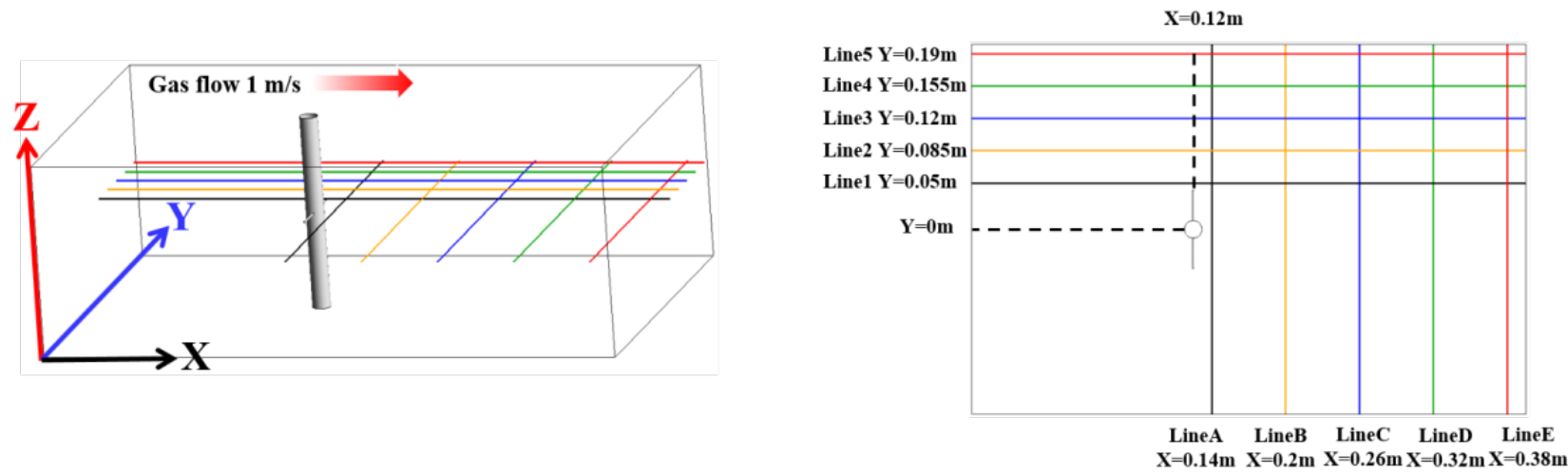

Fig. 6. Line distributions.

\section{Influence of Vortex on the Motion Behavior of a Single Particle with Different Injection Positions}

Fig. 6 shows the line setting position, where the lines are in different colors at the vertical direction of the needle tip from $Y=0.05-0.19 \mathrm{~m}$ and the back end of the polar line from $X=0.14-0.38 \mathrm{~m}$. The velocity and the turbulence intensity of each line at different positions are obtained via the postprocessing of the simulation results.

Fig. 7 shows the distributions of velocity and turbulence intensity at different positions. Graphs (a) and (b) show that when $X \leq 0.12 \mathrm{~m}$, the speed does not change much in most positions, which is almost the same as the set inlet velocity $\left(1 \mathrm{~m} \mathrm{~s}^{-1}\right)$, except the velocity near the edge of the dust collector has decreased slightly. When the distance of $X$ is 

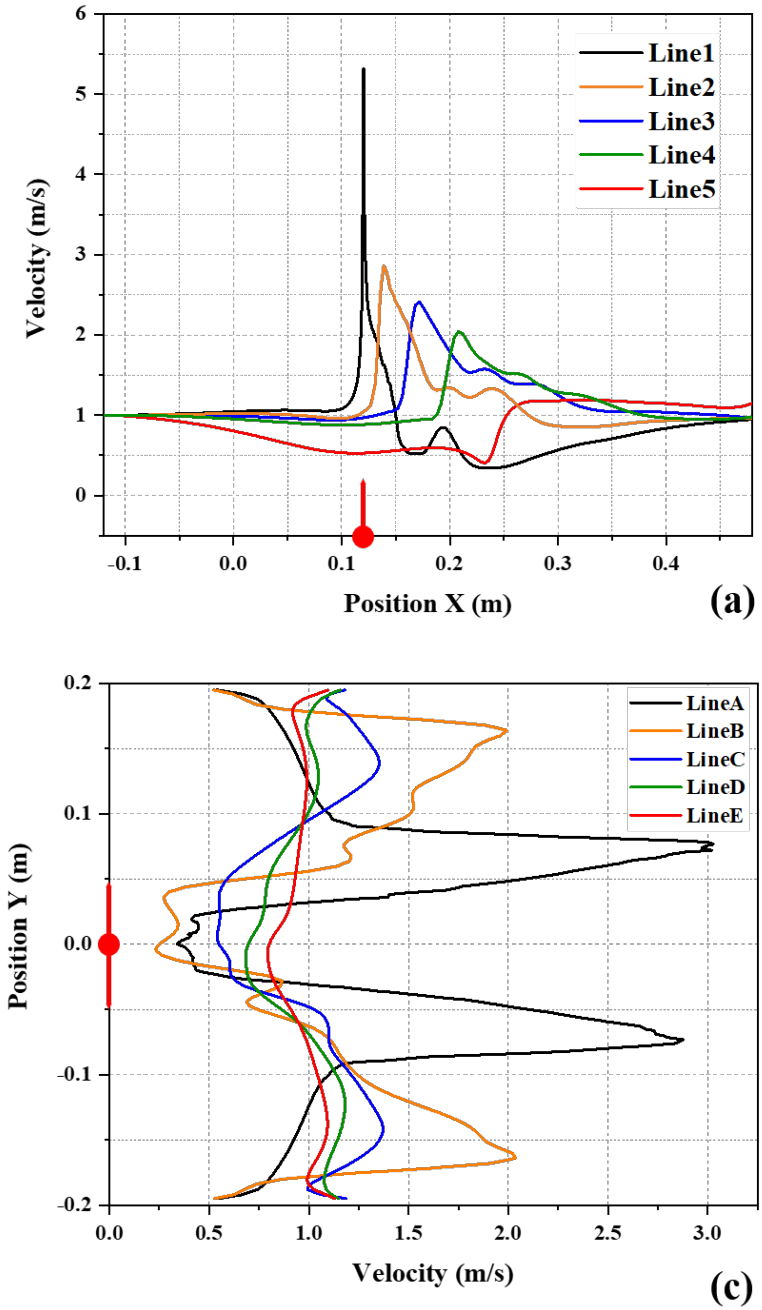
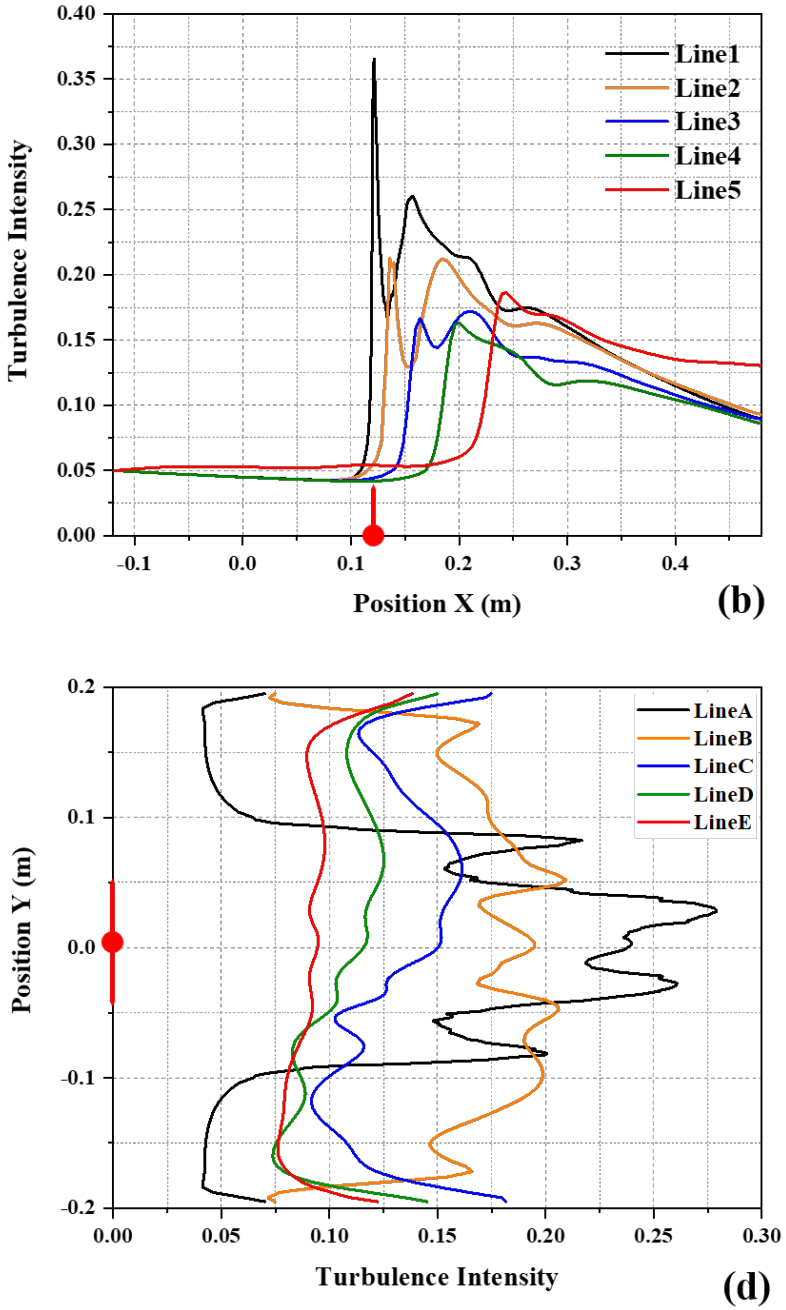

Fig. 7. Distributions of (a) velocity and (b) turbulence intensity at different positions and vertical direction of the (c) needle tip and (d) back end of the corona wire.

similar to the position of the needle tip (equal to $0.12 \mathrm{~m}$ ), the velocity closest to the needle tip dramatically increases from $1 \mathrm{~m} \mathrm{~s}^{-1}$ to $5.707 \mathrm{~m} \mathrm{~s}^{-1}$, and the velocity begins to drop significantly to $0.636 \mathrm{~m} \mathrm{~s}^{-1}$ after passing the discharge electrode. The same trend appears in all positions from Lines 2 to 5 , indicating that the rise and the fall of velocity occur as the distance from the tip of needle increases. Moreover, the maximum speed of each line decreases as its distance from the electrode increases. The turbulence intensity presents a similar trend with velocity (Fig. 7(b)) and does not drop immediately to the lowest point from the maximum value but decreases after some fluctuations. Unlike velocity, the turbulence intensity at the inlet position is not the same as the outlet in Figs. 7(a) and 7(b). These line distributions of velocity and turbulence intensity clearly point out that the ionic wind generated at the tip of needle greatly affects the flow field in almost half of the ESP channel.

Figs. 7(c) and 7(d) illustrate the velocity and turbulence intensity at the rear end of the needle corona wire to further understand the flow field characteristics behind the discharge electrode. A low-speed region is found just behind the electrode, where the position from $Y$ is between -0.05 and
$0.05 \mathrm{~m}$. Within this region, the velocity decreases to the minimum value $\left(0.253 \mathrm{~m} \mathrm{~s}^{-1}\right)$ when the position is close to the electrode, and the area with high velocity is reasonably found at the extended area behind the needle tip. These results clearly show an opposite trend to that of turbulence intensity, which increases sharply in the low-velocity region at the rear end of the corona wire, indicating that the needleshaped discharge electrode interferes with the gas flow. Therefore, the EHD flow generated in the ESP working process probably affects the particle capture.

The effect of EHD flow on the distribution of flow field and the formation of vortices is revealed in our previous work. However, the effect of EHD flow on particle trajectories is not involved. Therefore, in the present work, the trajectories of single particles with different particle sizes at two injection points are traced (Fig. 8). Large-sized particles (5, $10,20$, and $30 \mu \mathrm{m})$ are collected successfully when injected near the tip of the needle except for $1 \mu \mathrm{m}$ particles that escaped in a spiral path. These results indicate that when large-sized particles pass through the high-intensity discharge area, they are fully charged and driven quickly toward the dust collection plate due to the high-speed ionic wind generated 


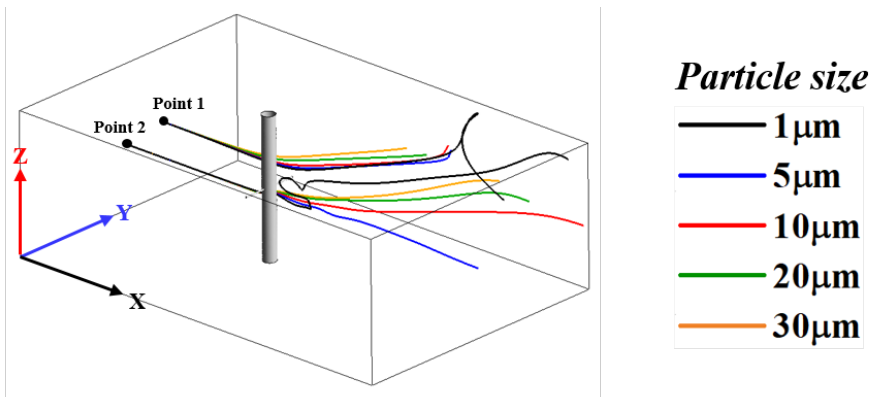

Injection point 1: Close to needle tip
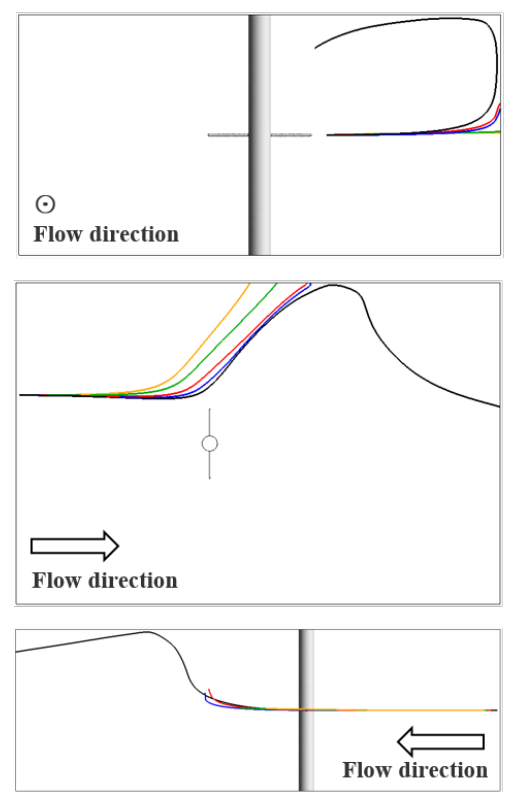

Injection point 2: Close to electrode wire
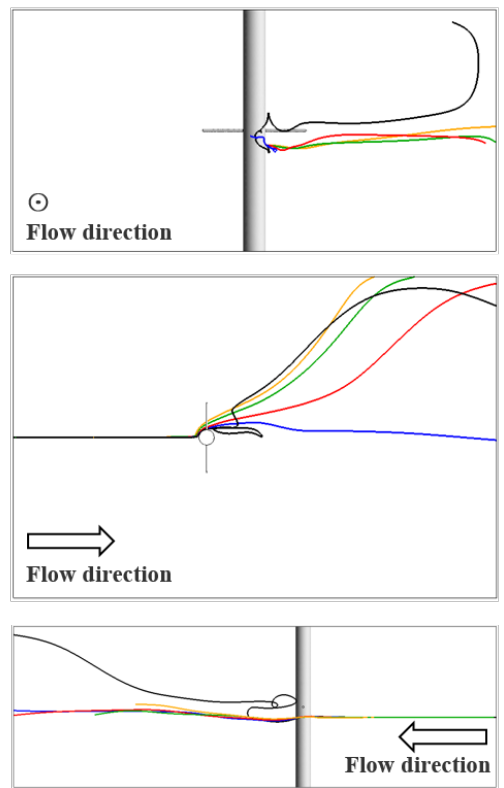

Fig. 8. Trajectories of a single particle with various particle diameters at different injection positions.

at the tip of the needle. Small particles are easily entrained by the vortex because of the spiral vortex generated by the collision of ionic wind and the collection wall surface. The movement trajectory of 5 and $10 \mu \mathrm{m}$ particles is also slightly deflected before reaching the dust collector. When the particles are injected in the middle position of the electrode, 1,5 , and $10 \mu \mathrm{m}$ particles escape, and only the 20 and $30 \mu \mathrm{m}$ particles are collected. Moreover, $5 \mu \mathrm{m}$ particles escape in a nearly straight trajectory without being affected by electrostatic precipitators. The movement trajectory of $1 \mu \mathrm{m}$ particles is very complex when passing around the electrode wire. The particles first move forward to a short distance, move backward to the electrode wire, and finally escape from the outlet. These phenomena may be caused by the low velocity and some vortices existing behind the electrode wire. Therefore, some meaningful suggestions are proposed. For example, the electrode geometry can be changed to make the discharge around the electrode uniform, reduce the dust collection blind area or optimize needle arrangement and pole distance, and reduce the formation of large-scale vortices. The staggered needle electrode can also be arranged. Results reveal that the trajectories of differently sized particles at different injection positions are affected by secondary flow, thereby helping to further understand the effect of EHD flow on particle capture.

\section{Multiple Effects of Ionic Wind on Particle Trapping}

The effect of EHD on particle capture is difficult to demonstrate in detail by only tracking the motion trajectory of a single particle. Thus, Fig. 9 clearly illustrates the top view of the distribution of particle velocity under different particle sizes and 5000 particles of each size from the entrance of ESP to further demonstrate the effect of secondary flow on particle motion. The distribution graphs reveal that the trajectory and the velocity of the particles have little change under different particle sizes when the particles do not pass the discharge electrode. After the particles pass through the discharge electrode, the particle velocity distribution shows that the particle velocities are accelerated under all particle sizes as the particles are close to the electrode. Two intuitive phenomena are observed on the particle trajectories under different particle sizes. (1) As the particle size decreases, the interspace at the back of the electrode decreases in size because the fine particles are easily affected by the low-velocity region at the rear end of the corona wire. (2) The spiral trajectory of the particles under all particle sizes is at the back part of the ESP channel because of the effect of secondary flow on particle movement. In particular, the trajectory of spiral motion becomes clear under small particle size. A heart-shaped pattern composed of particle trajectories is coincidentally 

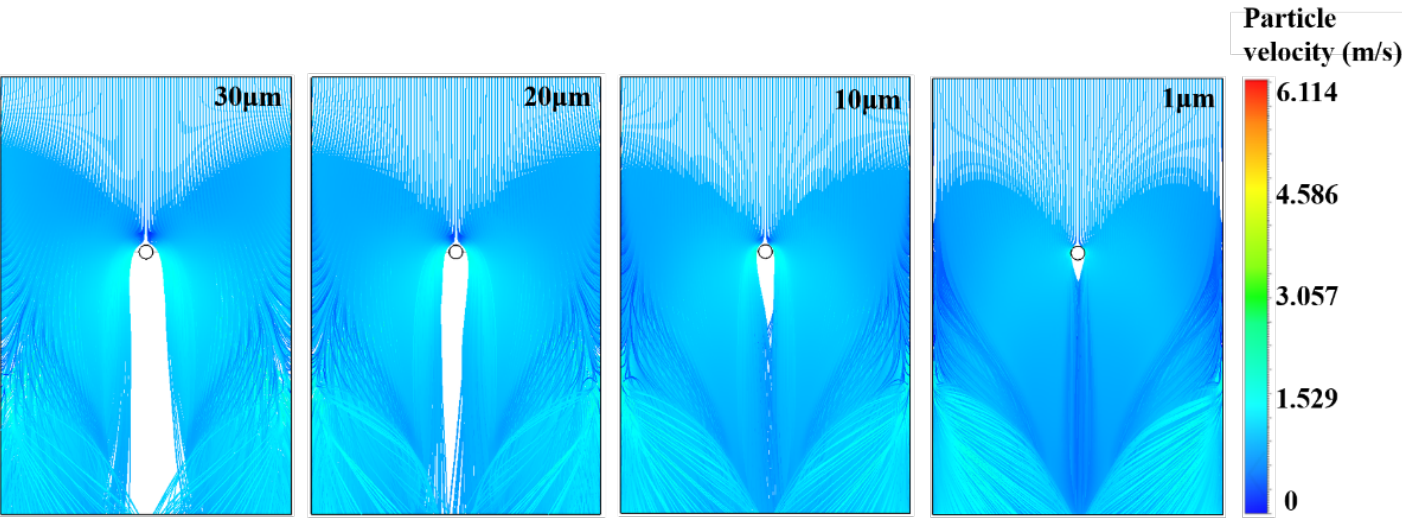

Fig. 9. Top view of the distribution of particle velocity under different particle sizes.

found in $1 \mu \mathrm{m}$ particles, indicating that the effect of EHD flow on particle motion varies with particle size.

The number of deposited particles on the collection surface significantly increases in front of the ESP channel and sharply decreases at the back of the ESP channel at an inlet velocity of $1 \mathrm{~m} \mathrm{~s}^{-1}$ (Fig. 10). Various surfaces with changed $X$ positions are presented to display the effect of EHD flow on particle collection performance under different particle sizes. Additional particles are deposited on the collection plate as the particle size increases from $1 \mu \mathrm{m}$ to $30 \mu \mathrm{m}$ because large particles are easily trapped with strong drag and electric forces, in which the penetration rate in the outlet surface decreases from $90.5 \%$ to $31.58 \%$. The distribution of the particle settling point on the collection and outlet surface shows that the ionic wind affects particle collection. In the collection surface, most particles are captured at the front of the ESP channel when the particles are accelerated forward to the collection plate by the high-speed ionic wind generated at the tip of the needle. However, the back end of the collection plate does not capture many particles under the whole particle size range, which may be because the vortex generated by the ionic wind drags the particles to escape from the back end of the ESP channel. The influence of EHD flow on particle collection can be explained in detail by observing the particle concentration in three surfaces with different $X$ positions. The distribution of the deposited particles on the surface with increased $X$ distance shows that the geometry of the needle electrode significantly affects the sedimentation distribution of the particles. The effect becomes clear as the particle size increases from $1 \mu \mathrm{m}$ to $30 \mu \mathrm{m}$ because the fine particles are easily trapped in the collection blind area at the rear end of the discharge electrode. The number of deposited particles is also mostly concentrated in four corners of the outlet surface, in which the positions are the same as those of the four large vortices found in the streamline distribution. This phenomenon becomes apparent with decreasing particle size. Moreover, the number of deposited particles in the middle of the outlet surface increases when the particle size decreases due to the movement of fine particles. This movement is easily affected by the regions with low velocity and high turbulence intensity at the rear end of the discharge electrode, as observed in our previous results. These results reveal that the effect of EHD flow on particle collection efficiency should not be underestimated, especially as the particle size decreases further.

The relationship among the injection position, residence time, and escape velocity of the particles under different sizes is shown in Fig. 11. The escape speed of the particles deposited onto the dust-collecting plate is set to $0 \mathrm{~m} \mathrm{~s}^{-1}$ (purple area) to determine the influence of different injection positions on particle collection. The particles are also uniformly injected from the ESP inlet at the same time.

First, $30 \mu \mathrm{m}$ particles are successfully captured when injected in most parts of the injected surface except for particles injected in the middle surface, which may be because the particles are too far away from the needle tip when passing through the electrode, thereby resulting in insufficient charge and escape from the ESP. At the same time, the particle residence time distribution of $30 \mu \mathrm{m}$ particles demonstrates that a long residence time has a high escape probability of particles. The maximum residence time of $30 \mu \mathrm{m}$ particles is 0.724 s. Second, the escape velocity distributions of the particles in different injection positions show that the particles escape from the ESP when injected in most positions as the particle size decreases from $30 \mu \mathrm{m}$ to $1 \mu \mathrm{m}$. With the decrease in particle size, the longest particle residence time and the fastest particle escape velocity increase from $0.724 \mathrm{~s}$ to $0.92 \mathrm{~s}$ and from $1.51 \mathrm{~m} \mathrm{~s}^{-1}$ to $1.62 \mathrm{~m} \mathrm{~s}^{-1}$, respectively, because fine particles are more susceptible to secondary flow. Finally, the relationship among the injection position of $1 \mu \mathrm{m}$ particles, residence time, and escape velocity distribution shows the following interferences of secondary flow on fine particle capture. (1) After $1 \mu \mathrm{m}$ particles are injected in a horizontal position with the discharge electrode, a relatively slow particle escape velocity and the longest particle residence time are observed because of the complex turbulence behind the discharge electrode. (2) When fine particles are injected near the needle tip, the particles escape rapidly and stay for a short time because of the high-speed ionic wind near the needle tip and the four large spiral vortices generated by the interaction with the collection wall. Both phenomena are not conducive to fine-particle capture. The relationship among the injection position, residence time, and escape velocity of the particles is not observed in previous research. The present study helps in understanding the effect of ionic wind on particle motion behavior. 

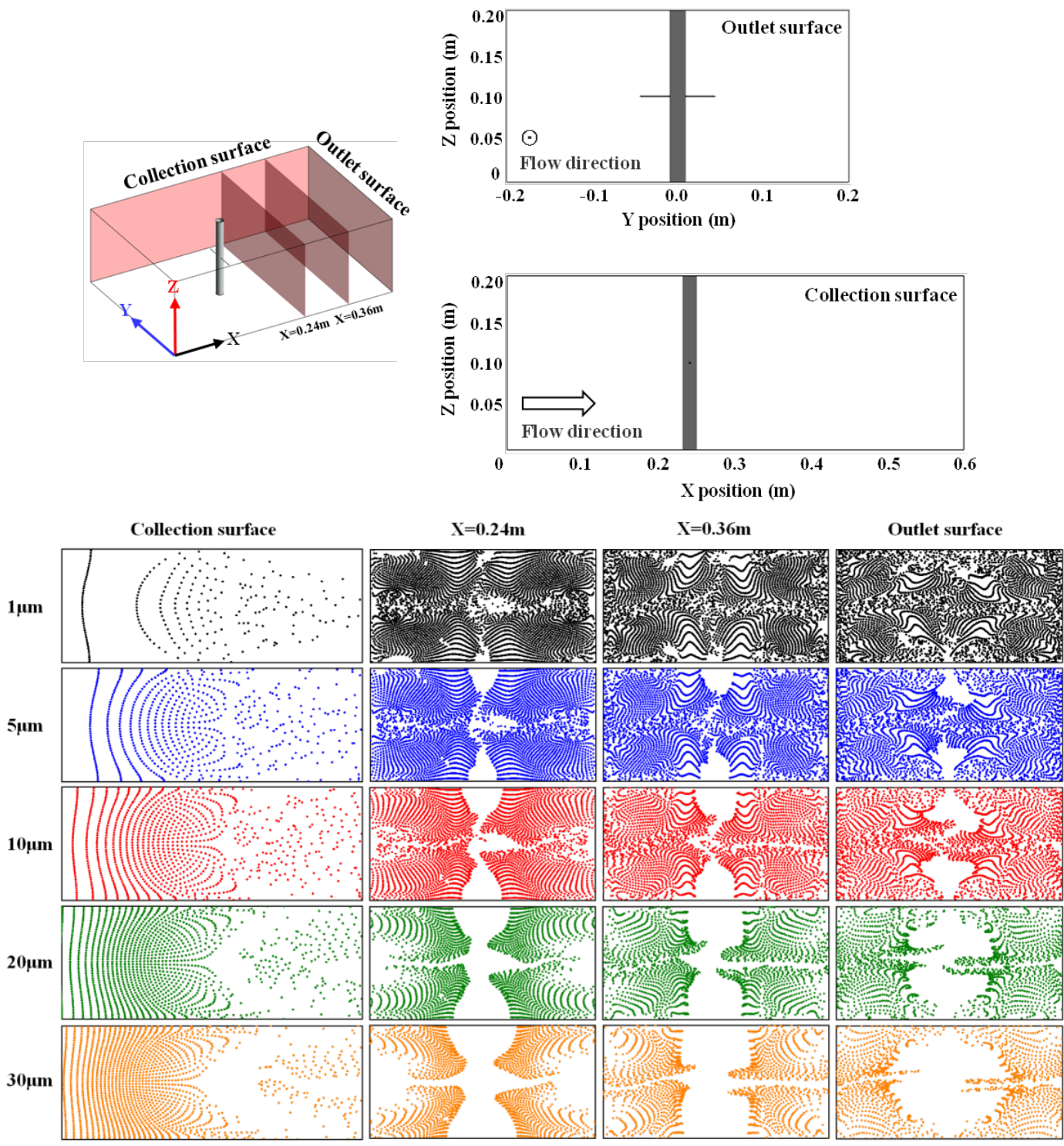

Fig. 10. Distribution of 5000 deposited particles on various surfaces under different particle sizes at an inlet velocity of $1 \mathrm{~m} \mathrm{~s}^{-1}$.

\section{CONCLUSION}

This research modeled a 3D single-needle-plate ESP and analyzed its flow field in detail, including the influence of the secondary flow on particle collection for various particle sizes. Our results indicate that numerical simulation shows great potential as a tool for examining the effect of complex flow fields on particle behavior in ESPs. The main conclusions are as follows.

1. The velocity of the ionic wind along the needle tip with the highest discharge intensity reached $9.028 \mathrm{~m} \mathrm{~s}^{-1}$ with an applied voltage of $-60 \mathrm{kV}$ and an inlet velocity of $1 \mathrm{~m} \mathrm{~s}^{-1}$. Flow field and streamline distribution analyses revealed that four large spiral ellipses were generated from the interaction between the high-speed ionic wind and the dust-collecting plate. A low-velocity blind area for dust collection was also observed to the rear of the discharge electrode.
2. When particles of different sizes were injected into the ESP, the ionic wind enhanced the collection of large particles by accelerating their migration to the dust collector. However, this wind adversely affected the collection of $1 \mu \mathrm{m}$ particles, which exhibited a penetration rate of $90.5 \%$ (versus $31.58 \%$ for $30 \mu \mathrm{m}$ particles). The distribution of the single-particle motion trajectories and the particle deposition points in the outlet and on the collection surface, which varied by particle size, illustrates the effect of the four large vortices and the low-velocity region to the rear of the corona wire on the fine fraction.

3. The relationship between the injection position, the residence time, and the escape velocity of the particles, demonstrated for the first time, further proves that the EHD flow significantly influences fine-particle collection. Our findings, which provide new insights into the effects of this factor, may assist the optimization of ESP design and operation. 


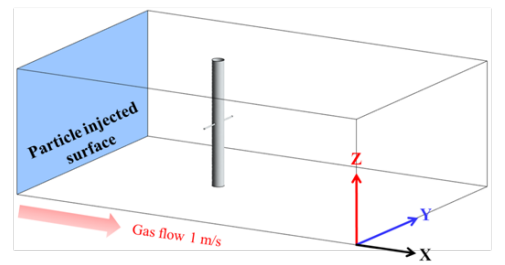

\section{Particle residence time}
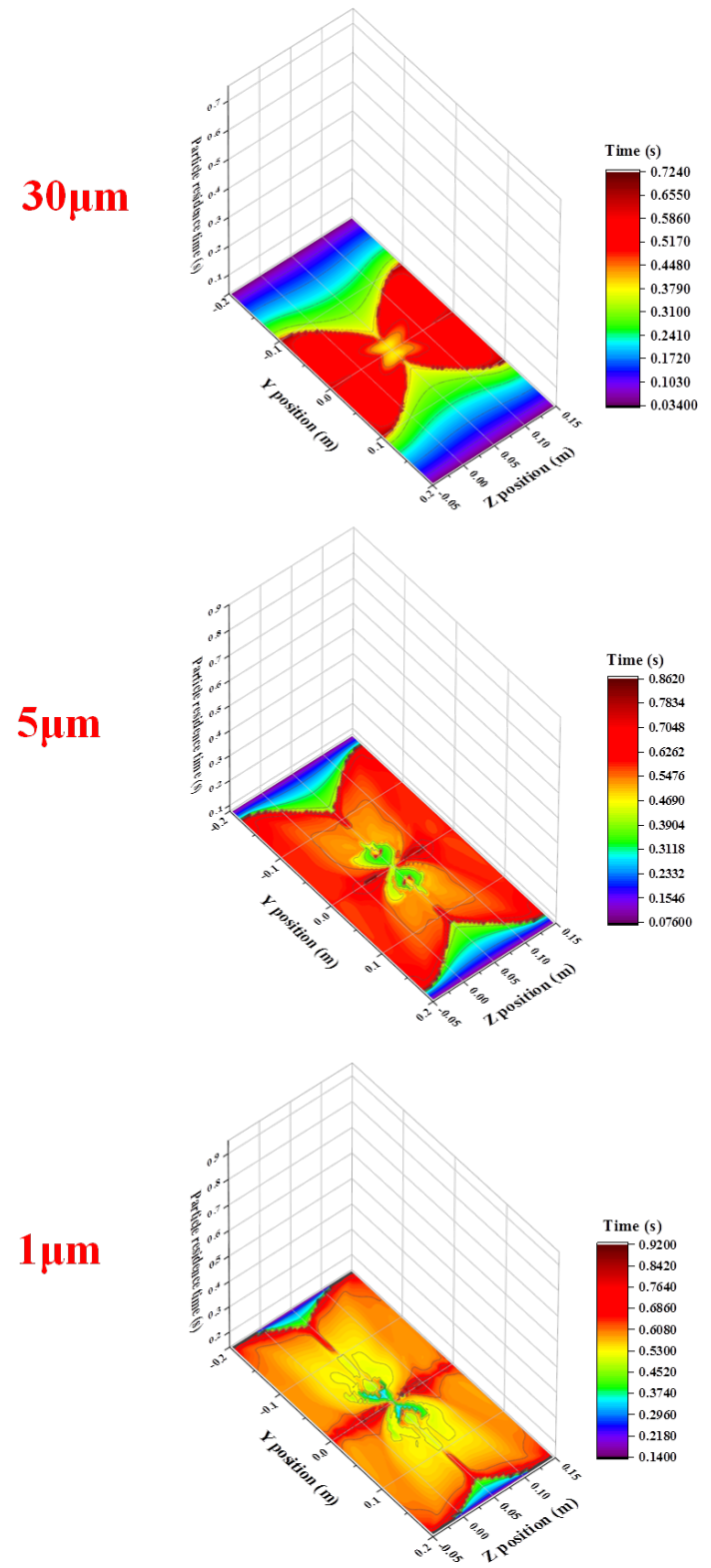

\section{Particle escape velocity}
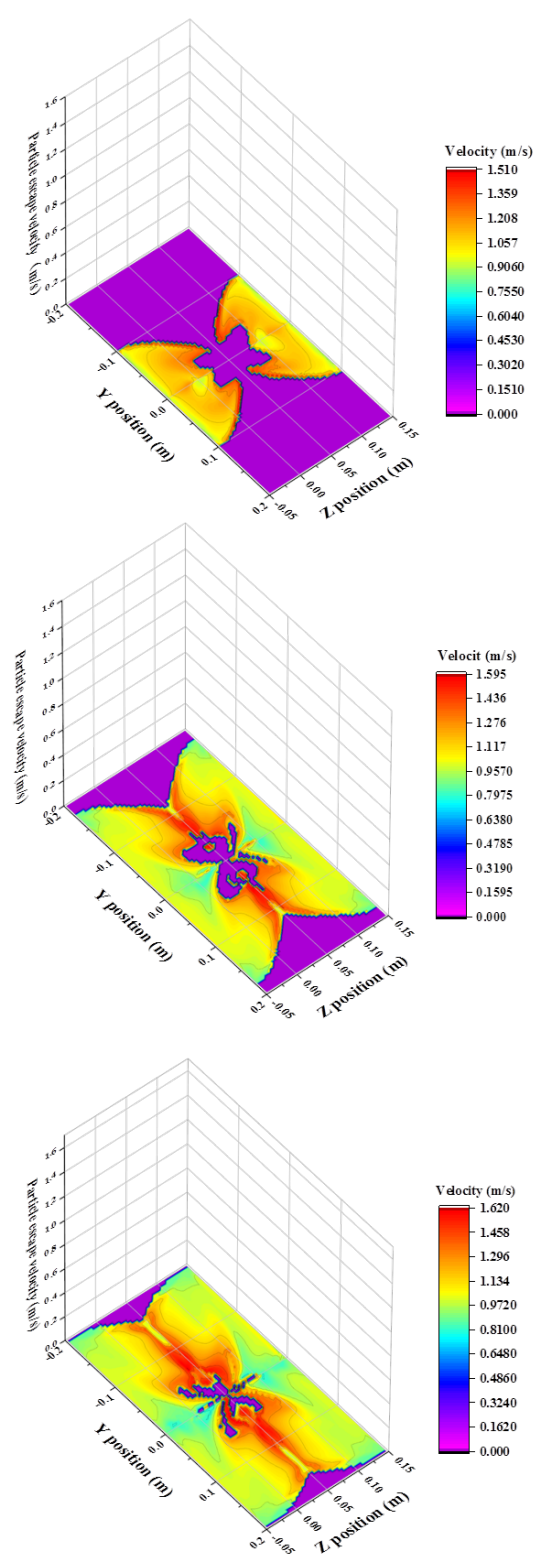

Fig. 11. Relationship among the injection position, residence time, and escape velocity of the particles under different sizes at an inlet velocity of $1 \mathrm{~m} \mathrm{~s}^{-1}$.

\section{ACKNOWLEDGEMENTS}

This work is supported by the Australia Research Council (ARC) Research Hub for Computational Particle Technology, Monash University (Australia), State Key Lab of Clean Energy Utilization and State Environmental Protection Engineering Center for Coal-Fired Air Pollution Control (China).

\section{REFERENCES}

Adamiak, K. (2013). Numerical models in simulating wireplate electrostatic precipitators: A review. J. Electrostat. 71: 673-680. https://doi.org/10.1016/j.elstat.2013.03.001 Chun, Y. (2006). Numerical modeling of wire electrohydrodynamic flow in a Wire-Plate ESP. Environ. 
Eng. Res. 11: 164-171. https://doi.org/10.4491/eer.2006. 11.3.164

Chun, Y.N., Chang, J.S., Berezin, A.A. and Mizeraczyk, J. (2007). Numerical modeling of near corona wire electrohydrodynamic flow in a wire-plate electrostatic precipitator. IEEE Trans. Dielectr. Electr. Insul. 14: 119124. https://doi.org/10.1109/TDEI.2007.302879

Czech, T., Sobczyk, A.T., Jaworek, A. and Krupa, A. (2012). Corona and back discharges in flue-gas simulating mixture. J. Electrostat. 70: 269-284. https://doi.org/10.1 016/j.elstat.2012.03.005

Dong, M., Zhou, F., Shang, Y. and Li, S. (2019). Numerical study on electrohydrodynamic flow and fine-particle collection efficiency in a spike electrode-plate electrostatic precipitator. Powder Technol. 351: 71-83. https://doi.org/ 10.1016/j.powtec.2019.03.046

Dong, M., Zhou, F., Zhang, Y., Shang, Y. and Li, S. (2018). Numerical study on fine-particle charging and transport behaviour in electrostatic precipitators. Powder Technol. 330: 210-218. https://doi.org/10.1016/j.powtec.2018.02. 038

Farnoosh, N., Adamiak, K. and Castle, G.S.P. (2010). 3-D numerical analysis of EHD turbulent flow and monodisperse charged particle transport and collection in a wire-plate ESP. J. Electrostat. 68: 513-522. https://doi.org/10.1016/j.elstat.2010.07.002

Farnoosh, N., Adamiak, K. and Castle, G.S.P. (2011). Three-dimensional analysis of electrohydrodynamic flow in a spiked electrode-plate electrostatic precipitator. $J$. Electrostat. 69: 419-428. https://doi.org/10.1016/j.elstat. 2011.06.002

Feng, Z., Long, Z., Cao, S. and Adamiak, K. (2018). Characterization of electrohydrodynamic (EHD) flow in electrostatic precitators (ESP) by numerical simulation and quantitative vortex analysis. J. Electrostat. 91: 70-80. https://doi.org/10.1016/j.elstat.2017.12.008

Fujishima, H., Morita, Y., Okubo, M. and Yamamoto, T. (2006). Numerical simulation of three-dimensional electrohydrodynamics of spiked-electrode electrostatic precipitators. IEEE Trans. Dielectr. Electr. Insul. 13: 160-167. https://doi.org/10.1109/TDEI.2006.1593414

Fujishima, H., Ueda, Y., Tomimatsu, K. and Yamamoto, T. (2004). Electrohydrodynamics of spiked electrode electrostatic precipitators. J. Electrostat. 62: 291-308. https://doi.org/10.1016/j.elstat.2004.05.006

Gao, W., Wang, Y., Zhang, H., Guo, B., Zheng, C., Guo, J., Gao, X. and Yu, A. (2020a). A numerical investigation of the effect of dust layer on particle migration in an electrostatic precipitator. Aerosol Air Qual. Res. 20: 166 179. https://doi.org/10.4209/aaqr.2019.11.0609

Gao, W., Wang, Y., Zhang, H., Guo, B., Zheng, C., Guo, J., Gao, X. and Yu, A. (2020b). Numerical simulation of particle migration in electrostatic precipitator with different electrode configurations. Powder Technol. 361: 238-247. https://doi.org/10.1016/j.powtec.2019.08.046

Guo, B.Y., Yang, D., Su, Y.B. and Yu, A.B. (2017). Process modelling of low temperature electrostatic precipitators. Powder Technol. 314: 567-576. https://doi.org/10.1016/j. powtec.2016.09.071
Guo, B.Y., Yang, S.Y., Xing, M., Dong, K.J., Yu, A.B. and Guo, J. (2013). Toward the development of an integrated multiscale model for electrostatic precipitation. Ind. Eng. Chem. Res. 52: 11282-11293. https://doi.org/10.1021/ie $303466 \mathrm{~g}$

Guo, B.Y., Yu, A.B. and Guo, J. (2014a). Numerical modeling of electrostatic precipitation: Effect of Gas temperature. J. Aerosol Sci. 77: 102-115. https://doi.org/1 0.1016/j.jaerosci.2014.07.009

Guo, B., Yu, A. and Guo, J. (2015). Numerical modelling of esp for design optimization. Procedia Eng. 102: 13661372. https://doi.org/10.1016/j.proeng.2015.01.268

Guo, B.Y., Guo, J. and Yu, A.B. (2014b). Simulation of the electric field in wire-plate type electrostatic precipitators. J. Electrostat. 72: 301-310. https://doi.org/10.1016/j.elst at.2014.05.005

Jaworek, A., Marchewicz, A., Sobczyk, A.T., Krupa, A. and Czech, T. (2018). Two-stage electrostatic precipitators for the reduction of $\mathrm{PM}_{2.5}$ particle emission. Prog. Energy Combust. Sci. 67: 206-233. https://doi.org/10.1016/j.pec s.2018.03.003

Kawakami, H., Sakurai, T., Ehara, Y., Yamamoto, T. and Zukeran, A. (2013). Performance characteristics between horizontally and vertically oriented electrodes EHD ESP for collection of low-resistive diesel particulates. $J$. Electrostat. 71: 1117-1123. https://doi.org/10.1016/j.elst at.2013.10.004

Moreau, E., Audier, P. and Benard, N. (2018). Ionic wind produced by positive and negative corona discharges in air. J. Electrostat. 93: 85-96. https://doi.org/10.1016/j.els tat.2018.03.009

Niewulis, A., Berendt, A., Podliński, J. and Mizeraczyk, J. (2013). Electrohydrodynamic flow patterns and collection efficiency in narrow wire-cylinder type electrostatic precipitator. J. Electrostat. 71: 808-814. https://doi.org/1 0.1016/j.elstat.2013.02.002

Niewulis, A., Podlinski, J. and Mizeraczyk, J. (2009). Electrohydrodynamic flow patterns in a narrow electrostatic precipitator with longitudinal or transverse wire electrode. J. Electrostatics. 67: 123-127. https://doi.org/10.1016/j.e lstat.2009.01.001

Park, S., Cvelbar, U., Choe, W. and Moon, S.Y. (2018). The creation of electric wind due to the electrohydrodynamic force. Nat. Commun. 9: 371. https://doi.org/10.1038/s414 67-017-02766-9

Podlinski, J., Niewulis, A. and Mizeraczyk, J. (2009). Electrohydrodynamic flow and particle collection efficiency of a spike-plate type electrostatic precipitator. $J$. Electrostatics. 67: 99-104. https://doi.org/10.1016/j.elst at.2009.02.009

Shen, H., Yu, W., Jia, H. and Kang, Y. (2018). Electrohydrodynamic flows in electrostatic precipitator of five shaped collecting electrodes. J. Electrostatics. 95: 61-70. https://doi.org/10.1016/j.elstat.2018.08.002

Soldati, A. (2000). On the effects of electrohydrodynamic flows and turbulence on aerosol transport and collection in wire-plate electrostatic precipitators. J. Aerosol Sci. 31: 293-305. https://doi.org/10.1016/S0021-8502(99)00055-5 Tang, L., Qu, J., Mi, Z., Bo, X., Chang, X., Anadon, L.D., 
Wang, S., Xue, X., Li, S., Wang, X. and Zhao, X. (2019). Substantial emission reductions from Chinese power plants after the introduction of ultra-low emissions standards. Nat. Energy 4: 929-938. https://doi.org/10.1038/s41560019-0468-1

Wang, J., Cai, Y.X., Li, X.H., Zhao, X.D., Wang, J., Shi, Y.F. and Shi, Y.X. (2017). Experimental investigation of high-power light-emitting diodes' thermal management by ionic wind. Appl. Therm. Eng. 122: 49-58. https://doi.org/10.1016/j.applthermaleng.2017.04.084

Wang, Y., Gao, W., Zhang, H., Huang, C., Luo, K., Zheng, C. and Gao, X. (2019). Insights into the role of ionic wind in honeycomb electrostatic precipitators. J. Aerosol Sci. 133: 83-95. https://doi.org/10.1016/j.jaerosci.2019.04.011

Yang, D., Guo, B., Ye, X., Yu, A. and Guo, J. (2019). Numerical simulation of electrostatic precipitator considering the dust particle space charge. Powder Technol. 354: 552-560. https://doi.org/10.1016/j.powtec. 2019.06.013

Yang, Z., Zheng, C., Li, Q., Zheng, H., Zhao, H. and Gao, X. (2020). Fast evolution of sulfuric acid aerosol activated by external fields for enhanced emission control. Environ. Sci. Technol. 54: 3022-3031. https://doi.org/10.1021/acs. est.9b06191

Zheng, C., Luo, C., Liu, Y., Wang, Y., Lu, Y., Qu, R.,
Zhang, Y. and Gao, X. (2020a). Experimental study on the removal of $\mathrm{SO}_{3}$ from coal-fired flue gas by alkaline sorbent. Fuel 259: 116306. https://doi.org/10.1016/j.fuel. 2019.116306

Zheng, C., Wang, Y., Liu, Y., Yang, Z., Qu, R., Ye, D., Liang, C., Liu, S. and Gao, X. (2019). Formation, transformation, measurement, and control of $\mathrm{SO}_{3}$ in coal-fired power plants. Fuel 241: 327-346. https://doi.org/10.1016/j.fuel. 2018.12.039

Zheng, C., Zheng, H., Shen, J., Gao, W., Yang, Z., Zhao, Z., Wang, Y., Zhang, H. and Gao, X. (2020b). Evolution of condensable fine particle size distribution in simulated flue gas by external regulation for growth enhancement. Environ. Sci. Technol. 54: 3840-3848. https://doi.org/10. 1021/acs.est.9b06569

Zhu, Y., Gao, M., Chen, M., Shi, J. and Shangguan, W. (2019). Numerical simulation of capture process of fine particles in electrostatic precipitators under consideration of electrohydrodynamics flow. Powder Technol. 354: 653-675. https://doi.org/10.1016/j.powtec.2019.06.038

Received for review, April 16, 2020

Revised, July 2, 2020 Accepted, July 19, 2020 